\title{
ON THE INTEGRATION OF NON-LINEAR PARABOLIC EQUATIONS BY IMPLICIT DIFFERENCE METHODS*
}

\author{
BY \\ MILTON E. ROSE \\ Office of Naval Research, ${ }^{1}$ Washington, D. C.
}

\begin{abstract}
The object of this paper is to investigate how solutions of mixed initialboundary value problems for a certain class of non-linear parabolic equations may be obtained by solving suitable implicit difference equations on a rectangular lattice and taking the limit of such solutions as the mesh of the lattice tends to zero.
\end{abstract}

We consider the non-linear ${ }^{2}$ parabolic differential equation

$$
\frac{\partial^{2} u}{\partial x^{2}}=F\left(x, t, u \frac{\partial u}{\partial x}, \frac{\partial u}{\partial t}\right)
$$

in the strip $0<t \leq T, 0<x<L$, with the initial condition

$$
-\beta^{0}(x) u(x, 0)=f_{0}(x)
$$

and the boundary conditions

$$
\begin{aligned}
\alpha^{1}(t) \frac{\partial u}{\partial x}(0, t)-\beta^{1}(t) u(0, t) & =f_{1}(t), \\
-\alpha^{2}(t) \frac{\partial}{\partial x} u(L, t)-\beta^{2}(t) u(L, t) & =f_{2}(t),
\end{aligned}
$$

assuming that the solution $u(x, t)$ is unique and exists with suitable regularity properties in the strip.

Introducing a family of rectangular lattices $\pi_{h}$ with mesh $(h, k)$ and non-negative weights $\omega_{1}, \omega_{2}$, we associate with (1) a family of implicit difference equations

$$
\begin{aligned}
\omega_{1} \nabla_{x}^{2} u(x, t)+\omega_{2} \nabla_{x}^{2} u(x, t-k) & \\
& =F\left[x, t, u(x, t), \omega_{1} \nabla_{x} u(x, t)+\omega_{2} \nabla_{x} u(x, t-k), \nabla_{x} u(x, t)\right]
\end{aligned}
$$

with corresponding difference equations approximating (1a) and (1b) [see (1.1)].

In the first part of this paper we prove the pointwise convergence, as we let $h, k \rightarrow 0$ in such a way that $k / h^{2}=\lambda$ is a fixed number, of the solutions $u^{h}(x, t)$ of the difference equations (2) to $u(x, t)$. We obtain estimates of the degree of convergence by methods suggested by arguments of Gèvrey [7] and Laasonen [8] for linear parabolic differential equations and related to [4], [5], [6] for solving elliptic equations using a maximum

${ }^{*}$ Received July 25, 1955; revised manuscript received October 3, 1955.

'This paper was written while the author was a member of the AEC Computing Facility, New York University. The author wishes to thank Prof. E. Isaacson and Drs. E. Bromberg and R. Richtmyer for their suggestions and criticism.

${ }^{2}$ For higher space dimensions our analysis would carry over with (1) replaced by

$$
\nabla^{2} u=F\left(t, x, y, \cdots ; u ; u_{\iota}, u_{x}, u_{y}, \cdots\right) \text {. }
$$

We will retain the form of our arguments suitable for extentions to higher dimensions. 
principle. Our proof of convergence imposes a restriction on the value of $\lambda$ [see (2.3)] which is stronger than the von Neumann criterion for linear equations.

An iteration method is then discussed for solving the implicit difference equations (2); a convergence proof is given which provides both an existence theorem for the solution of (2) for a fixed value of $h$ and estimates of the error at any stage of the iterations.

1. Statement of the problem. Consider the non-linear parabolic operator

$$
L[\phi] \equiv \frac{\partial^{2} \phi}{\partial x^{2}}-F\left(x, t, \phi, \frac{\partial \phi}{\partial x}, \frac{\partial \phi}{\partial t}\right) .
$$

$F(x, t, z, p, q)$ denotes a fixed continuous function of its variables for $(x, t)$ in a region $R$ in the $(x, t)$-plane and for all $z, p, q$. We assume that the partial derivative $F_{z}, F_{p}, F_{q}$ exist, are continuous, and satisfy the inequalities

$$
\begin{gathered}
0<a^{0} \leq F_{a} \leq A<\infty, \\
\left|F_{p}\right| \leq B<\infty, \\
0 \leq c^{0} \leq F_{a} \leq C<\infty,
\end{gathered}
$$

where $a^{0}, c^{0}, A, B$, and $C$ are fixed constants.

Let $R=R(T)$ be a domain in $R$ bounded by the coordinate lines $x=0, t=0$ and the lines $x=L, t=T$; the closure of $R$ will be denoted by $R^{\prime}$. The set composed of the segments $B_{0}(0<x<L, t=0), B_{1}(x=0,0<t \leq T)$ and $B_{2}(x=L, 0<t \leq T)$ will be denoted by $B=B(T)$ and called the boundary of $R^{\prime}$.

We define boundary operators $\Lambda_{0}, \Lambda_{1}, \Lambda_{2}$ by

$$
\begin{aligned}
& \Lambda_{0}[\varphi]=-\beta^{0}(x) \varphi(x, 0) \text { on } B_{0}, \\
& \Lambda_{1}[\varphi]=\alpha^{1}(t) \frac{\partial}{\partial x} \varphi(0, t)-\beta^{1}(t) \varphi(0, t) \text { on } B_{1} \\
& \Lambda_{2}[\varphi]=-\alpha^{2}(t) \frac{\partial}{\partial x} \varphi(L, t)-\beta^{2}(t) \varphi(L, t) \text { on } B_{2} ;
\end{aligned}
$$

here $\beta^{0}, \beta^{1}, \beta^{2}$ are continuous positive functions, $\alpha^{1}, \alpha^{2}$ are continuous non-negative functions on $B_{0}, B_{1}, B_{2}$. Let $f_{0}, f_{1}, f_{2}$ be fixed functions defined on $B_{0}, B_{1}, B_{2}$ respectively.

A mixed initial-boundary value problem $\odot$ may be formulated as follows: for fixed $T$, determine a function $u(x, t)$ defined in $R(T)$ with certain regularity properties satisfying the equation

$$
L[u]=0 \text { in } R
$$

and the initial and boundary conditions

$$
\Lambda_{i}[u]=f_{i} \quad \text { on } B_{i}, \quad i=0,1,2 .
$$

We will assume it to be known that this problem has at most one solution which exists with suitable regularity properties ${ }^{3}$ under appropriate regularity conditions on the operators $L, \Lambda$ and on the initial and boundary data.

3Specifically, we assume $u_{x x x x}, u_{x x t}, u_{t t}$ and lower order mixed partial derivatives exist and are continuous in $R$. 
Before describing a method for approximating the solution of problem $\odot$ by differences it will be convenient to introduce certain notations. Let $R_{h, k}^{i}$ be a rectangular lattice covering $R^{\prime}$ given by lines

$$
\begin{aligned}
x & =m h, \quad m & =0,1, \cdots, M, \\
t & =n k, \quad n & =0,1, \cdots, N,
\end{aligned}
$$

where $h=L / M, k=T / N$. A net point $P$ with coordinates $m h, n k$ will by denoted by $P_{m, n}$ or, simply, $(m, n)$. Let $\Delta_{n}$ denote the set of points with coordinates $(m, n)$ for $0<m<M$ and let $\Delta_{n}^{\prime}=\Delta_{n}+(0, n)+(M, n)$. A point of the set

$$
R_{h, k}=\sum_{n=1}^{N} \Delta_{n}
$$

will be called an interior point of $R_{h, k}$. A point of the set $B_{h, k}=R_{h, k}^{\prime}-R_{h, k}$ is a boundary point of $R_{h, k}^{\prime}$. Also, if $w$ is a function defined on $R_{h, k}^{\prime}$ we will write $w_{m, n}$ for $w\left(P_{m, n}\right)$. Furthermore, when $R_{h, k}^{\prime}$ is representative of a sequence of lattices obtained by letting $h, k$ approach zero in such a way that the ratio

$$
\frac{k}{h^{2}}=\lambda
$$

is fixed we write $R_{h, k}=R_{h}, B_{h, k}=B_{h}$, etc.

Next, let

$L_{h}\left[\varphi_{m, n}\right]=\omega_{1} \nabla_{x}^{2} \varphi_{m, n}+\omega_{2} \nabla_{x}^{2} \varphi_{m, n-1}-F\left[x_{n}, t_{n}, \varphi_{m, n}, \omega_{1} \nabla_{x} \varphi_{m, n}+\omega_{2} \nabla_{x} \varphi_{m, n-1}, \nabla_{\iota} \varphi_{m, n}\right]$, where

$$
\begin{aligned}
& \nabla_{x}^{2} \varphi_{m, n}=\frac{1}{h^{2}}\left(\varphi_{m+1, n}+\varphi_{m-1, n}-2 \varphi_{m, n}\right), \\
& \nabla_{z} \varphi_{m, n}=\frac{1}{2 h}\left(\varphi_{m+1, n}-\varphi_{m-1, n}\right), \\
& \nabla_{t} \varphi_{m, n}=\frac{1}{k}\left(\varphi_{m, n}-\varphi_{m, n-1}\right) .
\end{aligned}
$$

Here $\omega_{1} \geq 0, \omega_{2} \geq 0$ and $\omega_{1}+\omega_{2}=1$. Also, let

$$
\begin{aligned}
& \Lambda_{h, 0}\left[\varphi_{m, 0}\right] \equiv-\beta_{m}^{0} \varphi_{m, 0}, \quad 0 \leq m \leq M, \\
& \Lambda_{h, 1}\left[\varphi_{0, n}\right] \equiv \frac{\alpha_{n}^{1}}{h}\left(\varphi_{1, n}-\varphi_{0, n}\right)-\beta_{n}^{1} \varphi_{0, n}, \quad 0<n \leq N, \\
& \Lambda_{h, 2}\left[\varphi_{m, n}\right] \equiv \frac{\alpha_{n}^{2}}{h}\left(\varphi_{n-1, n}-\varphi_{n, n}\right)-\beta_{n}^{2} \varphi_{m, n} .
\end{aligned}
$$

The mixed initial-boundary value problem $P_{h}$ consists in finding a function $u^{h}$ defined on $R_{h}$ which satisfies the equation

$$
L_{\hbar}\left[u^{k}\right]=0 \text { in } R_{\hbar}
$$

and the initial and boundary conditions

$$
\Lambda_{h, i}\left[u^{h}\right]=f_{i}^{h} \text { on } B_{h, i},
$$

where $f_{i}^{h}$ is a given function on $B_{h, i}$. 
In Sec. 4 we describe an effective procedure for computing $u^{h}$ and obtain an existence and uniqueness theorem for problem $P_{h}$. In the next sections we derive sufficient conditions for solutions of $\mathcal{P}_{h}$ to approximate the solution of $P$ uniformly in $R^{\prime}$.

2. Estimates for linear difference operators. It will be sufficient to derive estimates for linear operators of the form

$$
\begin{aligned}
\chi_{n}\left[\varphi_{m, n}\right] \equiv \omega_{1} \nabla_{x}^{2} \varphi_{m, n}+\omega_{2} \nabla_{x}^{2} \varphi_{m, n-1} & -a_{m, n} \nabla_{1} \varphi_{m, n} \\
& -b_{m, n}\left[\omega_{1} \nabla_{x} \varphi_{m, n}+\omega_{2} \nabla_{x} \varphi_{m, n-1}\right]-c_{m, n} \varphi_{m, n},
\end{aligned}
$$

where

$$
0<a^{0} \leq a_{m, n} \leq A,\left|b_{m, n}\right| \leq B, 0 \leq c^{0} \leq c_{m, n} \leq C .
$$

We assume $h$ is so small, say $h<h_{0}$, that

$$
B h<B h_{0}<2 \theta,
$$

where $0<\theta<1$. Also, let $\lambda=k / h^{2}$.

Lemma 1. Let $\varphi$ be defined on $R_{h}^{\prime}$ with

and

$$
\chi_{h}[\varphi] \geq 0 \text { in } R_{h}
$$

Then, if

$$
\Lambda_{h, i}[\varphi] \geq 0 \quad \text { on } B_{h, i}, \quad i=0,1,2 .
$$

$$
\omega_{2} \lambda \leq \frac{a^{0}}{2},
$$

we have $\varphi \leq 0$ in $R_{h}^{\prime}$.

Proof. Let $R_{h}^{\gamma}, B_{h, i}^{\gamma}$ denote the subsets of $R_{h}, B_{h, i}$ respectively with points having $i$ coordinates less than or equal to $t_{v}$. Let $M_{h}^{n}[\varphi]=\max _{i} \max _{B_{A}, i}^{\prime} \varphi, i=0,1,2$. We first show that

$$
\max _{R_{A}} \varphi \leq M_{h}^{N}[\varphi] .
$$

We proceed by induction and suppose that (2.4) is true for $R_{h}^{\prime}$ and $B_{h, i}^{\prime}, 1 \leq \nu \leq N$, $i=0,1,2$ and that there exists at least one point, say $P_{m, n+1}$, of $\Delta_{n+1}$ for which $\varphi_{m, n+1}=$ $S_{.}>M_{h}^{n+1}[\varphi]$. From (2.1), (2.2) we obtain, since $\chi_{h}[\varphi] \geq 0$,

$$
\begin{aligned}
a_{m, n+1} \varphi_{m, n+1} \leq \Sigma_{m+1, n}\left(\varphi_{m+1, n+1}-\varphi_{m, n+1}\right) & +\Sigma_{m-1, n}\left(\varphi_{m-1, n+1}-\varphi_{m, n+1}\right) \\
& +\Gamma_{m+1, n} \varphi_{m+1, n}+\Gamma_{m, n} \varphi_{m, n}+\Gamma_{m-1, n} \varphi_{m-1, n} \\
& -k c_{m, n+1} \varphi_{m, n+1}
\end{aligned}
$$

where

$$
\begin{aligned}
\Sigma_{m \neq 1, n} & =\omega_{1} \lambda\left(1 \mp b_{m, n+1} \cdot \frac{h}{2}\right), \\
\Gamma_{m, n} & =\left(a_{m, n+1}-2 \lambda \omega_{2}\right), \\
\Gamma_{m=1, n} & =\omega_{2} \lambda\left(1 \mp b_{m, n+1} \cdot \frac{h}{2}\right) .
\end{aligned}
$$


By assumption,

so that

$$
h\left|b_{m, n+1}\right| \leq B h<2 \theta,
$$

$$
\Sigma_{m+1, n}>\omega_{1} \lambda(1-\theta) \geq 0
$$

and $\Sigma_{m+1, n}+\Sigma_{m-1, n}=2 \lambda \omega_{1}$. From 2.3 we have also $\Gamma_{m, n} \geq 0, \Gamma_{m+1, n} \geq 0$ and $\Gamma_{m+1, n}+$ $\Gamma_{m-1, n}+\Gamma_{m, n}=a_{m, n+1}>0$.

Suppose $\omega_{1} \neq 0$. Then, by assumption, there exists at least one point $P_{m^{\prime}, n+1}$ of $\Delta_{n+1}$ at which $\varphi_{m^{\prime}, n+1}=S$ and either $\left(\varphi_{m^{\prime}+1, n+1}-S\right)$ or $\left(\varphi_{m^{\prime}-1, n+1}-S\right)$ or both are less than zero. If $\omega_{2} \neq 0$,

$$
\max \left[\varphi_{m^{\prime}+1, n}, \varphi_{m^{\prime}, n}, \varphi_{m^{\prime}-1, n}\right] \leq M_{h}^{n}<S .
$$

In any case, therefore,

$$
a_{m^{\prime}, n+1} \cdot S<a_{m^{\prime}, n+1} \cdot S-k c_{m^{\prime}, n+1} \cdot S,
$$

which is impossible. For $n=1$, i.e., $R_{h, 1}^{1} B_{h, 1}^{1}(2.4)$ is obviously true and hence for $1 \leq n \leq N$.

We next show that $M_{h}^{n}[\varphi] \leq 0$. Suppose in fact $\varphi$ had a positive maximum at a point $Q$ of $B_{h, i}$. If $Q \subset B_{h, 0}$ we would have

$$
\Lambda_{h, 0}[\varphi(Q)]=-\beta^{0}(Q) \varphi(Q)=\sigma(Q) \geq 0,
$$

which is a contradiction if $\varphi(Q)>0$, since $\beta^{0}(Q)>0$. If $Q \subset B_{h, 1}$ or $B_{h, 2}$ and if $Q^{1}$ is the $h$-neighbor of $Q$ in $R_{h},(2.4)$ shows that

$$
\varphi\left(Q^{1}\right)-\varphi(Q) \leq 0 .
$$

In either case we would have

$$
0 \leq \sigma(Q)=\Lambda_{h,(1,2)}[\varphi(Q)] \leq-\beta^{(1,2)}(Q) \varphi(Q),
$$

which is a contradiction if $\varphi(Q)>0$. Thus $\varphi(Q) \leq 0$ for $Q \subset B_{h, i}, i=0,1,2$. This establishes the lemma.

From this lemma and the fact that $\chi_{h}, \Lambda_{h, i}$ are linear operators it follows that the only solution of the homogeneous problem is $\varphi \equiv 0$. The existence and uniqueness of a solution of the non-homogeneous problem follows directly from this fact.

Lemma 2. If $\varphi^{1}, \varphi^{2}$ are arbitrary functions on $R_{h}^{\prime}$ with

$$
\chi_{h}\left[\varphi^{1}\right] \leq-\left|\chi_{n}\left[\varphi^{2}\right]\right|
$$

and

$$
\Lambda_{h, i}\left[\varphi^{1}\right] \leq-\left|\Lambda_{h, i}\left[\varphi^{2}\right]\right|, \quad i=0,1,2,
$$

then

$$
\left|\varphi^{2}\right| \leq \varphi^{1} .
$$

The proof follows by letting $\varphi=\varphi^{2}-\varphi^{1}$ and applying Lemma 1 .

3. Proof of convergence. If $\varphi^{1}, \varphi^{2}$ are arbitrary functions and if $\varphi=\varphi^{1}-\varphi^{2}$, we have

$$
L_{h}\left[\varphi^{1}\right]-L_{h}\left[\varphi^{2}\right]=\chi_{n}[\varphi]
$$


where $\chi_{h}$ is given by (2.1) with

$$
a_{i, i}=F_{q}\left(x_{i}, t_{i}, z_{i, i}, \omega_{1} p_{i, i}+\omega_{2} p_{i, i-1}, q_{i, j}\right)
$$

and with $b_{i, i}=F_{p} c_{i j}=F_{z}$ evaluated at the same values of the arguments. Here

$$
\begin{aligned}
& z_{i, j}=\left\{\tau_{i, j} \varphi_{i, i}^{1}+\left(1-\tau_{i j}\right) \varphi_{i, i}^{2}\right\}, \\
& p_{i, j}=\left\{\tau_{i, j} \nabla_{x}\left[\varphi_{i, j}^{1}\right]+\left(1-\tau_{i, j}\right) \nabla_{x}\left[\varphi_{i, j}^{2}\right]\right\}, \\
& q_{i, j}=\left\{\tau_{i, j} \nabla_{t}\left[\varphi_{i, j}^{1}\right]+\left(1-\tau_{i, j}\right) \nabla_{t}\left[\varphi_{i, j}^{2}\right]\right\},
\end{aligned}
$$

with $0 \leq \tau_{i, i} \leq 1$

Let $v^{h}=u^{h}-u$, where $u^{h}, u$ are solutions of $\mathcal{P}_{h}, \mathcal{P}$ respectively. Then

$$
\chi_{h}\left[v^{h}\right]=-L_{h}[u] \text {. }
$$

Now set

$$
\left(m_{1}, m_{2}, m_{3}, m_{4}, m_{5}\right)=\max _{R^{\prime}}\left(\left|u_{x x x x}\right|,\left|u_{x x x}\right|,\left|u_{t t}\right|,\left|u_{x t}\right|,\left|u_{x x t}\right|\right)
$$

and put

$$
\Delta_{1}(h)=\frac{m_{1}}{12}+\frac{A h m_{3}}{3}
$$

and

$$
\Delta_{2}=\omega_{2}\left(m_{2}+A m_{5}\right)+\frac{B m_{4}}{2} .
$$

From

$$
\left|L_{h}[u]\right|=\left|L_{h}[u]-L[u]\right| \text {, }
$$

the usual Taylor expansion yields the estimate

$$
\left|L_{h}[u]\right| \leq h^{2} \Delta_{1}(h)+k \Delta_{2} \text {. }
$$

Similarly, if

$$
\alpha^{*}=\max _{i} \max _{B_{i}(T)}\left|\alpha^{i}\right|, \quad m_{6}=\max _{i} \max _{B_{i}(T)}\left|u_{x x}\right|
$$

we find

$$
\max _{i}\left|\Lambda_{h, i}[u]-\Lambda_{i}[u]\right| \leq h \alpha^{*} m_{6} \text {. }
$$

To obtain estimates for $v^{h}$ we introduce a comparison function $\Omega^{4}$ defined on $R^{\prime}$ for which

$$
\chi_{h}[\Omega] \leq-\frac{1}{2}
$$

and

$$
\Lambda_{h}[\Omega] \leq-\frac{1}{2}
$$

‘Explicit constructions of $\Omega$ are readily obtained following the construction in Bers [4]. 
Clearly, $\Omega \geq 0$ in $R^{\prime}$. Let

$$
\Omega^{\prime}=\max _{R_{\prime}} \Omega
$$

From (1.3), (3.1) we have

$$
\max _{i}\left|\Lambda_{h, i}\left[v^{h}\right]\right| \leq\left(\alpha^{*} m_{B}\right) h+\max _{i} \max _{B_{i}(T)}\left|f_{i}-f_{i}^{h}\right| .
$$

Let $\epsilon>0$ be chosen so that

$$
\epsilon \geq 2 \max \left[\left(h^{2} \Delta_{1}(h)+k \Delta_{2}\right),\left(\alpha^{*} m_{8} h+\max _{B(T)}\left|f-f^{h}\right|\right) .\right.
$$

Then

$$
\chi_{n}[\epsilon \Omega] \leq-\frac{\epsilon}{2} \leq-\left|\chi_{n}\left[v^{h}\right]\right|
$$

and

$$
\Lambda_{h, i}[\epsilon \Omega] \leq-\frac{\epsilon}{2} \leq-\left|\Lambda_{h, i}\left[v^{h}\right]\right|, \quad i=0,1,2 .
$$

Hence, from Lemma 2, we obtain

$$
\left|v^{n}\right| \leq \epsilon \Omega^{\prime},
$$

which furnishes a uniform estimate for $v^{k}$.

Assuming for the moment the existence of solutions $u^{h}$ of $\mathcal{P}_{h}$ we may restate our results in the

Theorem. Let problem $\odot$ be approximated by problems $\mathcal{P}_{h}$ in the sense that

$$
\max _{i} \max _{B_{i}(T)}\left|f_{i}-f_{i}^{h}\right|=\left\{\begin{array}{ccc}
\alpha^{*} O(h) & \text { if } & \alpha^{*} \neq 0, \\
O\left(h^{2}\right) & \text { if } & \alpha^{*}=0 .
\end{array}\right.
$$

Then, under the conditions detailed above, in particular, if $h, k \rightarrow 0$ in such a way that

$$
\lambda=\frac{k}{h^{2}} \leq \frac{a^{0}}{2 \omega_{2}},
$$

the solutions $u^{h}$ of $\mathcal{P}_{h}$ approximate the solution $u$ of problem $\mathcal{P}$ uniformly in $R^{\prime}(T)$; i.e.,

$$
\left|u-u^{h}\right|=\alpha^{*} O(h)+O\left(h^{2}\right)+O(k) .
$$

The condition on the mesh ratio $\lambda$ given here differs from the condition for stability in the von Neumann sense for linear equations, where one would expect stability for arbitrary $\lambda$ when $\omega_{2}<\frac{1}{2}$ (see [2], [3]). These facts raise the question whether our condition (3.2) may be weakened even in the non-linear case.

R. Richtmyer has given ${ }^{5}$ a convergence proof for the solution of the heat equation $u_{t}=u_{x x}$ by implicit difference equations of the type described here in which the von Neumann criterion is sufficient for his convergence arguments. From another viewpoint, condition (3.2) was imposed in order to obtain a maximum principle; F. John has shown

5Seminar in Numerical Analysis, Institute of Math. Sciences, New York University, 1954. 
[1], at least for explicit difference methods, convergence without using assumptions of positively weighted coefficients.

4. Construction of solutions of $P_{h}$ by iterations. In this section we shall describe an effective iteration method for calculating solutions of $P_{h}$ and obtain, at the same time, a constructive proof for the existence of solutions $u^{h}$ of $\mathcal{P}_{h}$.

Let

$$
\begin{aligned}
G_{i, i+1}\left(Z, \zeta_{1}, \zeta_{2} ;\right. & \left.Y_{i, j}\right)=\omega_{1}\left(\zeta_{1}+\zeta_{2}-2 Z\right)+\omega_{2}\left(Y_{i+1, i}+Y_{i-1, i}-2 Y_{i i}\right) \\
& -h^{2} F\left[x_{i}, t_{i+1}, Z, \frac{\omega_{1}}{2 h}\left(\zeta_{1}-\zeta_{2}\right)+\frac{\omega_{2}}{2 h}\left(Y_{i+1, i}-Y_{i-1, i}\right) \frac{Z-Y_{i, j}}{k}\right] .
\end{aligned}
$$

Let $\varphi^{0}$ satisfy the initial conditions

$$
\Lambda_{h, 0}\left[\varphi^{0}\right]=f_{0}^{h} \text { on } B_{0}
$$

and be otherwise given arbitrary on $R_{h}^{\prime}$.

Let $0_{i}$ be an ordering of the set $\Delta_{i}^{\prime}$. For $p_{i, i} \subset \Delta_{1}^{\prime}$ and, with

$$
H_{i, i}^{\delta}\left[Z ; \zeta^{1}, \zeta^{2} ; Z^{*}\right] \equiv Z+\delta G_{i, i}\left(Z, \zeta_{1}, \zeta_{2} ; Y_{i, i}\right)
$$

for $\delta>0$, set

$$
\varphi_{i, j}^{n+1}=H_{i, i}^{\delta}\left[(\varphi)_{i, j}^{n},(\varphi)_{i+1, j}^{n},(\varphi)_{i-1, i}^{n} ; \varphi_{i-1}\right],
$$

where

$$
(\varphi)_{i=1, i}^{n}=\varphi_{i=1, j}^{n+1}
$$

if $i+1$ precedes in the ordering $0_{i}$; otherwise

$$
(\varphi)_{i+1, i}^{n}=\varphi_{i+1, i}^{n}
$$

At boundary points $Q$ set

$$
\varphi^{n+1}(Q)=\frac{\alpha}{\beta h+\alpha}(\varphi)^{n}\left(Q^{\prime}\right)-\frac{h f^{h}(Q)}{\beta h+\alpha},
$$

$Q^{\prime}$ being the interior $h$-neighbor of $Q$.

Let $\varphi_{0} \equiv \varphi^{0}$ on $\Delta_{0}$ in (4.1). We shall show that

$$
\lim _{n \rightarrow \infty} \varphi_{i, 1}^{n} \rightarrow \varphi_{i, 1} \text {. }
$$

With $\varphi_{i, 1}$ considered as initial values on $\Delta_{1}^{\prime}$ calculate $\varphi_{i, 2}$ from (4.1), (4.2). Continuing in this manner we will obtain a function $\varphi_{i, j}$ defined in $R_{h}$ which, for $P_{i, i} \subset R_{h}$, satisfies

$$
G_{i, i+1}\left(\varphi_{i, i+1}, \varphi_{i+1, i+1}, \varphi_{i-1, i+1} ; \varphi_{i, j}\right)=0
$$

with

$$
\Lambda_{h, i}[\varphi]=f_{i}^{h} \text { on } B_{h, i}, \quad i=0,1,2,
$$

i.e. $\varphi_{i, j}$ is a solution, by construction, of $P_{h}$.

Finally we shall prove that, given any $\epsilon>0$, and for $N=T / k$, there exist integers $n_{1}, n_{2}, \cdots, n_{N}$ depending on $\epsilon$ such that

$$
\left|\varphi_{i, i}-\varphi_{i, j}^{n_{i}}\left[\varphi_{i-1}^{n_{i-1}}\right]\right|<\epsilon, \quad(i, j) \subset R_{h},
$$


where the notation $\varphi_{i, j}^{n_{i}}\left[\varphi_{i-1}^{n_{i}-1}\right]$ indicates the function obtained on $\Delta_{i}^{\prime}$ after $n_{i}$ iterations with "initial conditions" $\varphi_{i, j-1}^{n_{i}-1}$ on $\Delta_{i-1}^{\prime}$. (We will write $\varphi_{i, i}^{n}$ for $\varphi_{i, i}^{n}\left[\varphi_{i-1}\right]$ where no confusion is apt to arise.) This permits the effective computation of the solution numerically.

First of all, we have

$$
\begin{aligned}
\varphi_{i, i}^{n+1}-\varphi_{i, i}^{n}=\left(\frac{\partial H^{\delta}}{\partial z}\right)^{*}\left[(\varphi)_{i i}^{n}-(\varphi)_{i j}^{n-1}\right] & +\left(\frac{\partial H^{\delta}}{\partial \zeta_{1}}\right)^{*}\left[(\varphi)_{i+1, i}^{n}-(\varphi)_{i+1, i}^{n-1}\right] \\
& +\left(\frac{\partial H^{\delta}}{\partial \zeta_{2}}\right)^{*}\left[(\varphi)_{i-1, i}^{n}-(\varphi)_{i-1, i}^{n-1}\right]
\end{aligned}
$$

the asterisk indicating that the partial derivatives are evaluated at intermediate points

$$
\begin{array}{ll}
\tau_{i j}(\varphi)_{i+>, i}^{n}+\left(1-\tau_{i i}\right)(\varphi)_{i+1, i}^{n-1}, & \nu=-1,0,1 \\
& 0 \leq \tau_{i i} \leq 1
\end{array}
$$

At a boundary point $Q$ (from 4.2$)$

$$
\varphi^{n+1}(Q)-\varphi^{n}(Q)=\frac{\alpha}{\alpha+\beta h}\left[(\varphi)^{n}\left(Q^{\prime}\right)-(\varphi)^{n-1}\left(Q^{\prime}\right)\right]
$$

Let

$$
m_{i}^{n+1}=\max _{\Delta i}\left|\varphi_{i, i}^{n+1}-\varphi_{i, i}^{n}\right|
$$

and

$$
\mu_{i}^{n+1}=\max _{\left(\Delta^{\prime} i-\Delta_{i}\right)}\left|\varphi^{n+1}(Q)-\varphi^{n}(Q)\right|
$$

From (4.3) we have

$$
m_{i}^{n+1} \leq \max \left[m_{i}^{n}, \mu_{i}^{n}\right] \max _{\Delta_{i}}\left\{\left.\frac{\partial H_{i j}^{\delta}}{\partial z}\right|^{*}+\left|\frac{\partial H_{i j}^{\delta}}{\partial \zeta_{1}}\right|^{*}+\left|\frac{\partial H_{i i}^{\delta}}{\partial \zeta_{2}}\right|^{*}\right\} .
$$

Now

$$
\begin{aligned}
& \frac{\partial H_{i j}^{\delta *}}{\partial z}=1-\delta\left(2 \omega_{1}+h^{2} F_{z}^{*}+\frac{h^{2}}{k} F_{a}^{*}\right), \\
& \frac{\partial H_{i j}^{\delta *}}{\partial \zeta_{1}}=\delta \omega_{1}\left(1-\frac{h}{2} F_{p}^{*}\right), \\
& \frac{\partial H_{i j}^{\delta *}}{\partial \zeta_{2}}=\delta \omega_{1}\left(1+\frac{h}{2} F_{p}^{*}\right) .
\end{aligned}
$$

Choose

$$
\delta<\left(2 \omega_{1}+h^{2} A+\frac{h^{2}}{k} B\right)^{-1} .
$$

Then, since by assumption $1-(h / 2) F_{p}>0$, all these derivatives are non-negative and

$$
\left(\frac{\partial}{\partial Z}+\frac{\partial}{\partial \zeta_{1}}+\frac{\partial}{\partial \zeta_{2}}\right)\left(H_{i, j}^{\delta}\right)^{*}=1-\delta\left(h^{2} F_{z}^{*}+\frac{h^{2}}{k} F_{a}^{*}\right) \geq 0
$$


Let

$$
\rho=1-\delta h^{2}\left(A+k^{-1} B\right)
$$

Then (4.6) yields

$$
m_{i}^{n+1} \leq \rho \max \left[m_{i}^{n}, \mu_{j}^{n}\right] .
$$

From (4.4), however, we see that

$$
\mu_{i}^{n+1} \leq \frac{\alpha}{\alpha+\beta h} m_{i}^{n} \leq m_{i}^{n}
$$

so that

$$
m_{i}^{n+1} \leq \rho m_{i}^{n},
$$

or

$$
m_{i}^{n+1} \leq \rho^{n} m_{i}^{1}
$$

Thus

$$
\max _{\Delta \prime 1}\left|\varphi_{i, j}^{n+p}-\varphi_{i, j}^{n}\right| \leq \frac{m_{i}^{1} \rho^{n}}{1-\rho} .
$$

This implies the existence of a limit function $\varphi_{i, j}$,

$$
\varphi_{i, j}=\lim _{n \rightarrow \infty} \varphi_{i, j}^{n}, \quad j=1,2, \cdots, N,
$$

which is, by construction, a solution of $P_{h}$. The proof that this solution is unique follows directly.

Estimates similar to (4.7) hold for iterations based on a function $w_{i-1}$ taken as "initial values" on $\Delta_{i-1}^{\prime}$. Writing $\varphi_{i, i}=\varphi_{i, j}\left[\varphi_{i-1}\right]$, we have that

$$
\left|\varphi_{i j}^{n}\left[w_{i-1}\right]-\varphi_{i j}\right| \leq\left|\varphi_{i i}^{n}\left[w_{i-1}\right]-\varphi_{i j}\left[w_{i-1}\right]\right|+\left|\varphi_{i j}\left[w_{i-1}\right]-\varphi_{i j}\right| \text {. }
$$

Also

$$
\begin{aligned}
\varphi_{i, j}\left[w_{i-1}\right]-\varphi_{i, j}=\left(\frac{\partial H_{i j}^{\delta}}{\partial z}\right)^{*}\left(\varphi_{i, j}\left[w_{i-1}\right]-\varphi_{i, i}\right) & +\left(\frac{\partial H_{i j}^{\delta}}{\partial \zeta_{1}}\right)^{*}\left(\varphi_{i+1, i}\left[w_{i-1}\right]-\varphi_{i+1, j}\right) \\
& +\left(\frac{\partial H_{i j}^{\delta}}{\partial \zeta_{2}}\right)^{*}\left(\varphi_{i-1, i}\left[w_{i-1}\right]-\varphi_{i-1, i}\right) \\
& +\left(\frac{\partial H_{i j}^{\delta}}{\partial Y_{i j}}\right)^{*}\left(w_{i, i-1}-\varphi_{i, j-1}\right) \\
& +\left(\frac{\partial H_{i j}^{\delta}}{\partial Y_{i+1, j}}\right)^{*}\left(w_{i+1, i-1}-\varphi_{i+1, i-1}\right) \\
& +\left(\frac{\partial H_{i j}^{\delta}}{\partial Y_{i-1, j}}\right)^{*}\left(w_{i-1, i-1}-\varphi_{i-1, i-1}\right)
\end{aligned}
$$

the derivatives being evaluated at some intermediate point.

Let

$$
U_{i}\left[w_{i-1}\right]=\max _{\Delta^{\prime} i}\left|\varphi_{i, j}\left[w_{i-1}\right]-\dot{\varphi}_{i, i}\right|
$$


and

$$
V_{i}\left[w_{i-1}\right]=\max _{\Delta^{\prime} i-1}\left|w_{i, j-1}-\varphi_{i, j-1}\right|
$$

Now

$$
\begin{aligned}
\left(\frac{\partial H_{i i}^{\delta}}{\partial Y_{i j}}\right)^{*} & =\delta\left(\frac{h^{2}}{k} F_{i}^{*}-2 \omega_{2}\right), \\
\left(\frac{\partial H_{i i}^{\delta}}{\partial Y_{i+1, j}}\right)^{*} & =\delta \omega_{2}\left(1-\frac{h}{2} F_{p}^{*}\right), \\
\left(\frac{\partial H_{i j}^{\delta}}{\partial Y_{i-1, i}}\right)^{*} & =\delta \omega_{2}\left(1+\frac{h}{2} F_{p}^{*}\right) .
\end{aligned}
$$

Thus, from (4.8),

$$
U_{i}\left[w_{i-1}\right] \leq \rho U_{i}\left[w_{i-1}\right]+\kappa V_{i}\left[w_{i-1}\right]
$$

where

$$
\begin{aligned}
\kappa & =\frac{h^{2}}{k} A \quad \text { if } \quad \omega_{2} \leq \frac{h^{2}}{k} b^{0} \\
& =4 \omega_{2} \quad \text { if } \quad \omega_{2}>\frac{1}{2} \frac{h^{2}}{k} b^{0} .
\end{aligned}
$$

This may be written as

$$
U_{i}\left[w_{i-1}\right] \leq \frac{\kappa}{1-\rho} V_{i}\left[w_{i-1}\right] .
$$

Now, let $w_{i, j}=\varphi_{i, i}^{n i}$ where $n_{i}$ is some integer. From (4.7),

$$
V_{i}\left[\varphi_{j-1}^{n_{i-1}}\right] \leq \frac{\rho^{n_{j-1}}}{1-\rho} V_{i}\left[\varphi_{i-1}^{0}\right],
$$

so that, with

$$
W_{i}^{n}=\max _{\Delta^{\prime} i}\left|\varphi_{i, j}^{n}\left[\varphi_{i-1}^{n_{i}-1}\right]-\varphi_{i, j}\left[\varphi_{i-1}^{n_{i}-1}\right]\right|
$$

we have

$$
W_{i}^{n} \leq \rho^{n} W_{i}^{0} .
$$

Thus, from (4.7), (4.9), (4.10) we obtain

$$
\begin{aligned}
\left|\varphi_{i j}^{n}\left[\varphi_{i-1}^{n_{i-1}}\right]-\varphi_{i j}\right| & \leq W_{i}^{n}+U_{i}\left[\varphi_{i-1}^{n_{i}-1}\right] \\
& \leq \rho^{n} \cdot W_{i}^{0}+\frac{\kappa}{(1-\rho)^{2}} \cdot \rho^{n_{j-1}} V_{i}\left[\varphi_{i-1}^{0}\right] \quad j=1,2, \cdots, N .
\end{aligned}
$$

We may assume $V\left[\varphi_{0}^{0}\right]=0$. Let

$$
\Gamma_{1}=\max _{i=1,2, \cdots, N}\left|\frac{\kappa}{(1-\rho)^{2}} V_{i}\left[\varphi_{i-1}^{0}\right]\right|
$$


and assume $n_{1}, n_{2}, \cdots, n_{N}$ are so large that

$$
\max _{i=1,2, \cdots, N}\left|W_{i}^{0}\right| \leq \Gamma_{2} \text {. }
$$

Let

$$
\Gamma=\max \left[\Gamma_{1}, \Gamma_{2}\right]
$$

Then,

$$
\left|\varphi_{i, j}^{n}\left[\varphi_{i-1}^{n_{i-1}}\right]-\varphi_{i, j}\right| \leq \Gamma\left(\rho^{n}+\rho^{n_{i-1}}\right), \quad 1 \leq j \leq N
$$

and

$$
\left|\varphi_{i, j}^{n}-\varphi_{i, 1}\right| \leq \Gamma \rho^{n_{0}} \text {. }
$$

Hence, let $\epsilon>0$ be given. Let $n_{i}$ be determined, for example, such that

$$
\rho^{n_{i}} \leq \frac{j \epsilon}{2 N \Gamma}, \quad j \geq 1
$$

with

$$
\rho^{n_{0}} \leq \frac{\epsilon}{2 N \Gamma}
$$

Then

$$
\Gamma\left(\rho^{n_{i}}+\rho^{n_{i-1}}\right) \leq \frac{\epsilon}{2 N}(2 j-1)<\epsilon
$$

and $\Gamma \rho^{n_{0}}<\epsilon$.

This, then, furnishes a uniform approximation to the solution $\varphi_{i, j}$.in $R_{h}$, i.e., to the solution $u^{h}$ of $P_{h}$.

\section{Bibliography}

1. F. John, On integration of parabolic equations by difference methods, Communs. on Pure and Appl. Math. 5, 155-211 (1952)

2. R. P. Eddy, Stability in the numerical solution of initial value problems in partial differential equations, NOLM 10232, (1949)

3. G. G. O'Brien, M. A. Hyman, S. Kaplan, $A$ study of the numerical solution of partial differential equations, J. Math. Phys. 29, 223-251 (1951)

4. L. Bers, On mildly non-linear partial difference equations of elliptic type, J. Research Natl. Bur. Standards (1953)

5. S. Gerschgorin, Fehlerabschätzung für das Differenzenverfahren zur Lösung partieller Differentialgleichungen, Z. Angew. Math. Mech. 10, 373-382 (1930)

6. E. Batchelet, Über die numerische Auflösung von Randwertproblemen bei elliptischen partiellen Differentialgleichungen, ZAMP, 3, 165-193 (1952)

7. J. Gèvrey, Sur les équations aux derivées partielles du type parabolique, J. D. Math., Ser. 6,9 (1913); $10(1914)$

8. P. Laasonen, Über eine Methode zur Lösung der Wärmeleitungsgleichung, Acta Mathematica, 81 (1949) 